\title{
ON THE RELATION BETWEEN TWO LOCAL CONVERGENCE THEORIES OF LEAST-CHANGE SECANT UPDATE METHODS
}

\author{
JOSÉ MARIO MARTÍNEZ
}

\begin{abstract}
In this paper, we show that the main results of the local convergence theory for least-change secant update methods of Dennis and Walker (SIAM J. Numer. Anal. 18 (1981), 949-987) can be proved using the theory introduced recently by Martínez (Math. Comp. 55 (1990), 143-167). In addition, we exhibit two generalizations of well-known methods whose local convergence can be easily proved using Martínez's theory.
\end{abstract}

\section{INTRODUCTION}

Quasi-Newton ( $\mathrm{q}-\mathrm{N}$ ) methods have been widely used for a long time to solve systems of nonlinear equations (see [14]). Given the system $F(x)=0$, $F: \mathbb{R}^{n} \rightarrow \mathbb{R}^{n}$, these methods iterate according to

$$
x_{k+1}=x_{k}-B_{k}^{-1} F\left(x_{k}\right),
$$

where $B_{k+1}$ is obtained from $B_{k}$ using simple procedures which, usually, do not involve computation of derivatives of $F$. Sometimes it is also easy to obtain $B_{k+1}^{-1}$ (or a factorization of $B_{k}$ ) in a nonexpensive way, so that a great deal of computational work is saved.

Much research has been done on investigating the local convergence of quasiNewton methods (see [2, 3, 4, 9, 12, 14, 15, 20, 21, 28], etc.). Local convergence theorems assume that a solution $x_{*}$ of the system exists and, usually, that the Jacobian $J(x)$ satisfies a Hölder condition, and that $J\left(x_{*}\right)$ is nonsingular. Under these hypotheses it is usually proved that $x_{k}$ converges to $x_{*}$ if $x_{0}$ and $B_{0}$ are close to $x_{*}$ and $J\left(x_{*}\right)$, respectively. Often, superlinear convergence (or convergence at some "ideal" linear rate) can also be proved.

Different quasi-Newton methods differ in the way in which $B_{k+1}$ is obtained. However, most practical quasi-Newton algorithms share the characteristics of being "least-change secant update" (LCSU) methods (see [13, 14, $19,15])$. This means that $B_{k+1}$ satisfies a "secant equation" which guarantees that $B_{k+1}\left(x_{k+1}-x_{k}\right) \approx J\left(x_{k+1}\right)\left(x_{k+1}-x_{k}\right)$ with a minimum variation property

Received by the editor October 25, 1990 and, in revised form, May 30, 1991 and October 25, 1991.

1991 Mathematics Subject Classification. Primary 65H10.

Key words and phrases. Nonlinear systems, quasi-Newton methods, secant methods, least-change secant update methods.

This work was supported by FAPESP (Proc. 90/3724-6), FINEP, CNPq, and FAEP-UNICAMP. 
relative to some norm on the matrix space. By the minimum variation requirement and the secant equation, the sequence of matrices exhibit a phenomenon known as "Bounded Deterioration" $[9,4,12,14]$. This property guarantees that the matrices $B_{k}$ stay in a given neighborhood of $J\left(x_{*}\right)$, providing the essential arguments for proving local convergence at a linear rate. In view of the secant equation, it is possible to apply the necessary and sufficient condition for superlinear convergence of Dennis and Moré [12].

Prior to the work of Dennis and Walker [15], a new proof was required for each different algorithm. The Dennis-Walker theory had the merit of unifying most of them. So, the first and second methods of Broyden [1], the Sparse Broyden (or Schubert) method [3, 32], the PSB method [31], the method of Greenstadt [19], the DFP method [8, 17], the BFGS method [2, 16, 18, 33], the sparse symmetric method of Marwil [29] and Toint [34], etc. are all algorithms for which local and superlinear convergence can be proved using the DennisWalker theory.

Dennis and Walker also considered methods where the iteration formula is given by

$$
x_{k+1}=x_{k}-\left(C\left(x_{k}\right)+A_{k}\right)^{-1} F\left(x_{k}\right)
$$

or

$$
x_{k+1}=x_{k}-\left(C\left(x_{k}\right)+A_{k}\right) F\left(x_{k}\right)
$$

and established sufficient convergence conditions for them. In (1.1) (resp. (1.2)) $C\left(x_{k}\right)$ is a "computed part" of $J\left(x_{k}\right)$ (resp. $J\left(x_{k}\right)^{-1}$ ) and $J\left(x_{k}\right)-C\left(x_{k}\right)$ (resp. $\left.J\left(x_{k}\right)^{-1}-C\left(x_{k}\right)\right)$ is difficult to compute. So, $A_{k}$ is intended to be an approximation of $J\left(x_{k}\right)-C\left(x_{k}\right)$ (resp. $J\left(x_{k}\right)^{-1}-C\left(x_{k}\right)$ ). The main application of algorithms of the form (1.1) or (1.2) are secant augmentations of the GaussNewton method for nonlinear least squares problems (see [14, 10]).

In the decade of the 80's some new methods appeared which preserve the structure of the true Jacobian in a way not covered by the Dennis-Walker theory. We have mainly in mind the family of Partitioned Quasi-Newton methods [20, $21,22,23,35]$, the family of superlinear methods with direct secant updates of matrix factorizations [25, 5, 27], and the Secant Finite Difference method of Dennis and $\mathrm{Li}[11]$.

The Dennis-Walker theory does not apply to Partitioned q-N methods or to methods based on direct updating of factorizations because in these methods the matrices $B_{k}$ are not directly updated using variational principles. Instead, minimum variation is applied to underlying parameters which lie in a different space. The reasons why the Dennis-Li method is not covered by the DennisWalker theory are to be explained later in the present paper.

Motivated by the desirability of looking at all these methods under a common framework, Martinez [28] developed a new convergence theory which includes the new methods developed in the 80's, as well as all the classical methods covered by the Dennis-Walker theory. Martinez's theory is fairly simple, and the sufficient conditions it states for local convergence are easy to verify in practical situations. However, by the time Martinez's paper appeared, it was not clear if this theory was in fact more general than the theory of Dennis and Walker or if, on the contrary, there could exist algorithms whose convergence behavior could 
be explained by the Dennis-Walker theory but not by the Martinez theory. In this paper we answer this question.

In $\S 2$ of this paper we survey the part of Martinez's theory which is relevant for the purposes of the present research. The original theory is more general because it considers the use of $\mathrm{q}-\mathrm{N}$ approximations as preconditioners for inexact-Newton procedures, but this extension is not relevant for our present purposes. Accordingly, we consider essentially algorithms of the form

$$
x_{k+1}=x_{k}-\varphi\left(x_{k}, E_{k}\right)^{-1} F\left(x_{k}\right),
$$

where $\varphi$ is continuous and $E_{k} \in X$, a finite-dimensional linear space. Local linear convergence of (1.3) depends on three assumptions. Superlinear convergence (or convergence at an "ideal" rate $r_{*}$ ) is achieved if, asymptotically, a secant-type equation is satisfied.

In $\S 3$ we consider the "direct least-change secant update" methods of Dennis and Walker, and we prove local "ideal" convergence for these methods, showing that they are particular cases of the general algorithm of $\S 2$. In $\S 4$ the same work is done with respect to the "inverse least-change secant update" methods. Both direct and inverse least-change secant update methods are considered in their fixed-scale version and their iteratea-scale form. In $\S 5$ we introduce a potentially useful generalization of the Secant-Finite Difference method of Dennis and $\mathrm{Li}$, and in $\S 6$ we generalize the method of Hart and Soul for boundary value problems, and we prove local superlinear convergence using Martinez's theory.

Notation. Throughout this paper, $|\cdot|$ denotes an arbitrary norm on $\mathbb{R}^{n}$ and its subordinate matrix norm. $\left\{e_{1}, \ldots, e_{n}\right\}$ is the canonical basis of $\mathbb{R}^{n}$.

\section{SURVEy of MARTínez'S local CONVERGENCE THEORY}

In this section we survey the main results of Martinez's theory [28]. Considering the objectives of this paper, we state these results in their quasi-Newton version, instead of the inexact-Newton version, which is more general.

The problem is to solve

$$
F(x)=0
$$

for $x \in \Omega, \Omega$ an open and convex set of $\mathbb{R}^{n}, F: \Omega \rightarrow \mathbb{R}^{n}, F \in C^{1}(\Omega)$. We denote $J(x) \equiv F^{\prime}(x)$ for all $x \in \Omega$.

Let $X$ be a finite-dimensional linear space. For all $x, z \in \Omega$, let $\|\cdot\|_{x z}$ be a norm on $X$, associated with some scalar product $\langle,\rangle_{x z}$.

The projection operator onto a set $\mathscr{C} \subset X$ with respect to $\|\cdot\|_{x z}$ will be denoted by $P_{\mathscr{C}}, x z$.

For all $x, z \in \Omega$, let $V(x, z) \subset X$ be a linear manifold. Let $D \subset X$ be an open set. Let $\varphi: \Omega \times D \rightarrow \mathbb{R}^{n \times n}$ be a continuous function.

For arbitrary $x_{0} \in \Omega, E_{0} \in D$, and $B_{0}=\varphi\left(x_{0}, E_{0}\right)$, we consider the sequence generated by

$$
x_{k+1}=x_{k}-B_{k}^{-1} F\left(x_{k}\right) \text {, }
$$

where

$$
\begin{gathered}
B_{k+1} \in\left\{\varphi\left(x_{k}, E_{k}\right), \varphi\left(x_{k+1}, E_{k}\right), \varphi\left(x_{k}, E_{k+1}\right), \varphi\left(x_{k+1}, E_{k+1}\right)\right\}, \\
E_{k+1}=P_{k}\left(E_{k}\right),
\end{gathered}
$$

and $P_{k} \equiv P_{V\left(x_{k}, x_{k+1}\right), x_{k} x_{k+1}}$. 
In [28] only the choice

$$
B_{k+1}=\varphi\left(x_{k+1}, E_{k+1}\right)
$$

is considered. This is the choice used in most practical methods. However, we will see here that the linear convergence result that is proved in [28] for (2.5) extends trivially to (2.3).

We now state the assumptions that ensure that the sequence generated by (2.2) $-(2.4)$ is locally well defined and convergent to some solution of (2.1). The sequence generated by $(2.2)-(2.4)$ may not be well defined for three reasons: (a) some $x_{k}$ does not belong to $\Omega$, (b) some $E_{k}$ does not belong to $D$, or (c) $\varphi\left(x_{k}, E_{k}\right)$ is singular.

If $x_{k+1}=x_{k}$ we must have $F\left(x_{k}\right)=0$. In this case we say that the sequence stops at $x_{k}$, obtaining an exact solution of $(2.1)$. Hence, we are only going to consider the case where $x_{k+1} \neq x_{k}$ for all $k=0,1,2, \ldots$.

Assumption 1. Let $x_{*} \in \Omega$ be such that $F\left(x_{*}\right)=0$ and $J\left(x_{*}\right)$ is nonsingular, and let $L, p>0$ be such that

$$
\left|J(x)-J\left(x_{*}\right)\right| \leq L\left|x-x_{*}\right|^{p}
$$

for all $x \in \Omega$. This implies (see [4]) that

$$
\left|F(z)-F(x)-J\left(x_{*}\right)(z-x)\right| \leq L|z-x| \sigma(x, z)^{p}
$$

for all $x, z \in \Omega$, where $\sigma(x, z)=\max \left\{\left|x-x_{*}\right|,\left|z-x_{*}\right|\right\}$.

Assumption 2. Assume that there exists $E_{*} \in D$ such that $\varphi\left(x_{*}, E_{*}\right)$ is nonsingular and

$$
\left|I-\varphi\left(x_{*}, E_{*}\right)^{-1} J\left(x_{*}\right)\right| \leq r_{*}<1
$$

Assumption 3. Let $\|\cdot\|$ be a fixed norm on $X$, associated with the scalar product $\langle$,$\rangle , and let c_{1} \geq 0$ be a constant. Assume that for all $x, z \in \Omega$, there exists $E=E(x, z) \in V(x, z)$ such that

$$
\left\|E-E_{*}\right\| \leq c_{1} \sigma(x, z)^{p} .
$$

Assumption 4. There exist $q>0$ and $c_{2} \geq 0$ such that for all $x, z \in \Omega$, and for all $E \in X$,

$$
\|E\|_{x z} \leq\left[1+c_{2} \sigma(x, z)^{q}\right]\|E\|
$$

and

$$
\|E\| \leq\left[1+c_{2} \sigma(x, z)^{q}\right]\|E\|_{x z} .
$$

Throughout this section, we assume that Assumptions 1-4 are satisfied.

Let us state first a local linear convergence theorem.

Theorem 2.1. Let $r \in\left(r_{*}, 1\right)$. There exist $\varepsilon=\varepsilon(r)$ and $\delta=\delta(r)$ such that, if $\left|x_{0}-x_{*}\right| \leq \varepsilon$ and $\left\|E_{0}-E_{*}\right\| \leq \delta$, then the sequence generated by (2.2), (2.3), and (2.4) is well defined, converges to $x_{*}$, and satisfies

$$
\left|x_{k+1}-x_{*}\right| \leq r\left|x_{k}-x_{*}\right|
$$


for all $k=0,1,2, \ldots$ Moreover, $\left\|E_{k}\right\|,\left|B_{k}\right|$, and $\left|B_{k}^{-1}\right|$ are uniformly bounded.

Proof. The proof of this result is based on bounded deterioration arguments $($ see $[9,4])$ and follows by an adaptation of the proof of Theorem 3.2 of [28].

We include a detailed proof for the sake of completeness.

Let $\varepsilon_{1}, \delta_{1}>0$ be such that $\|E\|,|\varphi(x, E)|$, and $\left|\varphi(x, E)^{-1}\right|$ are uniformly bounded and

$$
\left|x-\varphi(x, E)^{-1} F(x)-x_{*}\right| \leq r\left|x-x_{*}\right|
$$

for all $x, E$ such that $\left\|x-x_{*}\right\| \leq \varepsilon_{1}$ and $\left\|E-E_{*}\right\| \leq \delta_{1}$. The existence of $\varepsilon_{1}$ and $\delta_{1}$ is guaranteed by Theorem 3.1 of [28]. By Corollary 3.1 of [28] there exists $c>0$ such that

$$
\left\|P_{x z}(E)-E_{*}\right\| \leq\left\|E-E_{*}\right\|+c\left|x-x_{*}\right|^{s}
$$

for all $x, E$ such that $\left\|x-x_{*}\right\| \leq \varepsilon_{1}$ and $\left\|E-E_{*}\right\| \leq \delta_{1}$, where $P_{x z} \equiv P_{V(x, z), x z}$ and $s=\min \{p, q\}$. Let $\delta \in\left(0, \delta_{1}\right]$ and $\varepsilon \in\left(0, \varepsilon_{1}\right]$ be such that

$$
\delta+c \varepsilon^{s} /\left(1-r^{s}\right)<\delta_{1}
$$

We prove that for all $k=0,1,2, \ldots$

(i) $x_{k+1}$ is well defined,

(ii) $\left|x_{k+1}-x_{*}\right| \leq r\left|x_{k}-x_{*}\right|$,

(iii) $\left|x_{k+1}-x_{*}\right| \leq r^{k+1} \varepsilon$,

(iv) $\left\|E_{k+1}-E_{*}\right\| \leq \delta+c \varepsilon^{s} \sum_{j=0}^{k} r^{s j}$.

We prove (i)-(iv) by induction on $k$. For $k=0$, (i)-(iv) follow trivially from (2.13) and (2.14). Assume now the inductive hypothesis for $k-1$. Thus,

$$
\left\|E_{k}-E_{*}\right\| \leq \delta+c \varepsilon^{s} \sum_{j=0}^{k-1} r^{s j} \leq \delta+c \varepsilon^{s} \sum_{j=0}^{\infty} r^{s j} \leq \delta+\frac{c \varepsilon^{s}}{1-r^{s}}<\delta_{1} .
$$

Similarly, $\left\|E_{k+1}-E_{*}\right\|<\delta_{1}$. But, by the inductive hypotheses, $\left|x^{k}-x^{*}\right| \leq$ $r^{k} \varepsilon \leq \varepsilon$ and $\left|x^{k-1}-x_{*}\right| \leq r^{k-1} \varepsilon \leq \varepsilon$. So, by (2.3) and (2.13), $x_{k+1}$ is well defined and satisfies (ii). (iii) follows trivially from (ii), and (iv) follows from (2.14) and the inductive hypothesis.

Finally, we deduce from (ii) and (iii) that $\lim _{k \rightarrow \infty} x_{k}=x_{*},\left\|x_{k}-x_{*}\right\| \leq \varepsilon$, and $\left\|E_{k}-E_{*}\right\| \leq \delta_{1}$ for all $k=0,1,2, \ldots$. Then, by (2.3) and Theorem 3.1 of [28], $\left|B_{k}\right|$ and $\left|B_{k}^{-1}\right|$ are uniformly bounded.

The following theorem states a Dennis-Moré condition for convergence of the sequence at the ideal rate $r_{*}$.

Theorem 2.2. Assume that the sequence generated by (2.2), (2.5), and (2.4) is well defined and that, for some $r \in\left(r_{*}, 1\right)$, we have

$$
\left|x_{k+1}-x_{*}\right| \leq r\left|x_{k}-x_{*}\right|
$$

for $k=0,1,2, \ldots$. Assume that

$$
\lim _{k \rightarrow \infty} \frac{\left|\left[\varphi\left(x_{k}, E_{k}\right)-\varphi\left(x_{*}, E_{*}\right)\right]\left(x_{k+1}-x_{k}\right)\right|}{\left|x_{k+1}-x_{k}\right|}=0 .
$$

Then

$$
\varlimsup \frac{\left|x_{k+1}-x_{*}\right|}{\left|x_{k}-x_{*}\right|}=\varlimsup \frac{\left|\left[I-\varphi\left(x_{*}, E_{*}\right)^{-1} J\left(x_{*}\right)\right]\left(x_{k}-x_{*}\right)\right|}{\left|x_{k}-x_{*}\right|} \leq r_{*} .
$$


Proof. Observe first that

$$
\begin{aligned}
& \frac{\left|x_{k}-x_{*}-B_{*}^{-1} F\left(x_{k}\right)\right|}{\left|x_{k}-x_{*}\right|}-\frac{\left|\left(B_{*}^{-1}-B_{k}^{-1}\right) F\left(x_{k}\right)\right|}{\left|x_{k}-x_{*}\right|} \\
& \quad \leq \frac{\left|x_{k+1}-x_{*}\right|}{\left|x_{k}-x_{*}\right|} \leq \frac{\left|x_{k}-x_{*}-B_{*}^{-1} F\left(x_{k}\right)\right|}{\left|x_{k}-x_{*}\right|}+\frac{\left|\left(B_{*}^{-1}-B_{k}^{-1}\right) F\left(x_{k}\right)\right|}{\left|x_{k}-x_{*}\right|},
\end{aligned}
$$

where $B_{*}=\varphi\left(x_{*}, E_{*}\right)$.

The proof follows repeating the arguments used in the proof of Theorem 3.4 of [28]. The hypothesis $\left|x_{Q}^{k}-x^{*}\right| \leq r\left|x^{k}-x^{*}\right|$ in [28, Theorem 3.4] is satisfied since, in our case, $x_{Q}^{k}=x_{k+1}$. So, the desired result follows from (2.18) and formula (3.36) of [28].

The following theorems report the behavior of the sequence $\left\{E_{k}\right\}$ under the hypothesis of linear convergence.

Theorem 2.3. Suppose that (2.15) holds for all $k=0,1,2, \ldots$. Then $\left\|E_{k}\right\|$ is uniformly bounded and

$$
\lim _{k \rightarrow \infty}\left\|E_{k+1}-E_{k}\right\|=0 \text {. }
$$

Proof. By (2.10), (2.11), (2.9), and (2.15) we prove, using Lemma 3.1 of [28], that

$$
\left\|E_{k+1}-E_{*}\right\| \leq\left(1+c_{4}\left|x_{k}-x_{*}\right|^{q}\right)\left\|E_{k}-E_{*}\right\|+c_{3}\left|x_{k}-x_{*}\right|^{p},
$$

where $c_{3}$ and $c_{4}$ are positive constants. Then, by Lemma 3.3 of [12], $\left\|E_{k}-E_{*}\right\|$ is uniformly bounded. So, $\left\|E_{k}\right\|$ is uniformly bounded. To prove (2.19), we repeat the proof of Theorem 3.3 of [28]. In fact, by the uniform boundedness of $\left\|E_{k}-E_{*}\right\|$, we have, by (2.20), that there exists $c_{5}>0$ such that

$$
\left\|E_{k+1}-E_{*}\right\| \leq\left\|E_{k}-E_{*}\right\|+c_{5}\left|x_{k}-x_{*}\right|^{s}
$$

for all $k=0,1,2, \ldots$. So, by $(2.15)$ and (2.21),

$$
\left\|E_{k+j}-E_{*}\right\| \leq\left\|E_{k}-E_{*}\right\|+c_{6}\left|x_{k}-x_{*}\right|^{s}
$$

for all $k, j=0,1,2, \ldots$, where $c_{6}=c_{5} /\left(1-r^{s}\right)$. Therefore, by the uniform boundedness of $\left\|E_{k}-E_{*}\right\|$ and $\left\|x_{k}-x_{*}\right\|$, there exists $c_{7}>0$ such that

$$
\left\|E_{k+j}-E_{*}\right\|^{2} \leq\left\|E_{k}-E_{*}\right\|^{2}+c_{7}\left|x_{k}-x_{*}\right|^{s} \text {. }
$$

Inequality (2.23) is inequality (3.25) of [28]. Now, the proof of Theorem 3.3 of [28] can be completely reproduced replacing the references to Theorem 3.2 by (2.15) and the reference to (3.25) by (2.23).

Theorem 2.4. Assume the hypotheses of Theorem 2.3. Suppose that there exists a closed set $\Gamma \subset \mathbb{R}^{n} \times X$ such that $\left(x_{k}, E_{k}\right) \in \Gamma \subset \Omega \times D$ for all $k=0,1,2, \ldots$. Then

$$
\lim _{k \rightarrow \infty}\left|\varphi\left(x_{k+1}, E_{k+1}\right)-\varphi\left(x_{k}, E_{k}\right)\right|=0 .
$$

Proof. From the convergence of $\left\{x_{k}\right\}$, the uniform boundedness of $\left\{\left\|E_{k}\right\|\right\}$, and the existence of the closed set $\Gamma$, we deduce that there exists a compact set $\Gamma^{\prime} \subset \Omega \times D$ such that $\left(x_{k}, E_{k}\right) \in \Gamma^{\prime}$ for all $k=0,1,2, \ldots$. Then, $(2.24)$ follows from the uniform continuity of $\varphi$ on $\Gamma^{\prime}$.

In the following theorem it is proved that, if (2.15) is assumed, convergence at the ideal rate $r_{*}$ follows from an "asymptotic secant condition." 
Theorem 2.5. Suppose that (2.15) holds and $\left(x_{k}, E_{k}\right) \in \Gamma \subset \Omega \times D$ for all $k=0,1,2, \ldots$, where $\Gamma$ is a closed subset in $\mathbb{R}^{n} \times X$. Assume that

$$
\lim _{k \rightarrow \infty} \frac{\left|\left[\varphi\left(x_{k+1}, E_{k+1}\right)-\varphi\left(x_{*}, E_{*}\right)\right]\left(x_{k+1}-x_{k}\right)\right|}{\left|x_{k+1}-x_{k}\right|}=0 .
$$

Then,

$$
\varlimsup \frac{\left|x_{k+1}-x_{*}\right|}{\left|x_{k}-x_{*}\right|}=\varlimsup \frac{\left|\left[I-\varphi\left(x_{*}, E_{*}\right)^{-1} J\left(x_{*}\right)\right]\left(x_{k}-x_{*}\right)\right|}{\left|x_{k}-x_{*}\right|} \leq r_{*} .
$$

Proof. The desired result follows from Theorems 2.2 and 2.4.

Theorem 2.6. There exist $\varepsilon, \delta>0$ such that, if $\left|x_{0}-x_{*}\right| \leq \varepsilon,\left\|E_{0}-E_{*}\right\| \leq \delta$, and (2.25) holds, the sequence generated by (2.2), (2.5), and (2.4) converges to $x_{*}$ and satisfies (2.17).

Proof. The convergence is proved in Theorem 2.1. Using bounded deterioration, we prove that, taking $\delta$ small enough, all the $E_{k}$ 's belong to a closed ball contained in $D$. So, the thesis follows from Theorem 2.5 (see Theorem (3.5) of [28]).

\section{LOCAL CONVERGENCE OF THE DIRECT LEAST-CHANGE SECANT UPDATE METHODS}

We are now going to use the preceding theorems to prove the main results of Dennis and Walker [15]. The first method introduced by Dennis and Walker [15] is the "fixed-scale least-change secant update method." In order to introduce this method, assume that $F \in C^{1}(\Omega)$ as in (2.1) and that, for all $x \in \Omega$,

$$
J(x)=C(x)+N(x),
$$

where $C(x)$ is continuous in $\Omega$.

Let $\mathscr{A} \subset \mathbb{R}^{n \times n}$ be a linear manifold, and denote the subspace parallel to $\mathscr{A}$ by $\mathscr{S}$.

For all $s, y \in \mathbb{R}^{n}$, we define

$$
Q(y, s)=\left\{A \in \mathbb{R}^{n \times n} \mid A s=y\right\} .
$$

Let $\|\cdot\|_{*}$ be a norm on $\mathbb{R}^{n \times n}$, associated with some scalar product. The projection operator onto a set $\mathscr{C} \subset \mathbb{R}^{n \times n}$ with respect to $\|\cdot\|_{*}$ will be denoted $P_{\mathscr{C}, *}$.

For any $A \in \mathbb{R}^{n \times n}$ and $\mathscr{C} \subset \mathbb{R}^{n \times n}$ we define

$$
\operatorname{dist}_{*}(A, \mathscr{C})=\inf _{B \in \mathscr{C}}\left\{\|A-B\|_{*}\right\} .
$$

For all $x, z \in \Omega$, we choose $y=y(x, z) \in \mathbb{R}^{n}$. Define

$$
\begin{aligned}
V(x, z)=\{A \in \mathscr{A} \mid & \operatorname{dist}_{*}(A, Q(y(x, z), z-x)) \\
& \left.\leq \operatorname{dist}_{*}\left(A^{\prime}, Q(y(x, z), z-x)\right) \text { for all } A^{\prime} \in \mathscr{A}\right\} .
\end{aligned}
$$

Dennis and Schnabel [13] proved that $V(x, z)$ defined by (3.3) is a linear manifold and that its parallel subspace is $\mathscr{S} \cap Q(0, z-x)$.

The fixed-scale least-change secant update method proposed by Dennis and Walker [15] consists of the iteration

$$
x_{k+1}=x_{k}-B_{k}^{-1} F\left(x_{k}\right),
$$


where

$$
B_{k+1} \in\left\{C\left(x_{k}\right)+A_{k}, C\left(x_{k+1}\right)+A_{k}, C\left(x_{k}\right)+A_{k+1}, C\left(x_{k+1}\right)+A_{k+1}\right\}
$$

and

$$
A_{k+1}=P_{V\left(x_{k}, x_{k+1}\right), *} \text {. }
$$

Now let us state the assumptions which ensure that the sequence generated by (3.4)-(3.6) is well defined and converges to a solution of $(2.1)$. The first one is Assumption 1 of $\S 2$.

Assumption DF2. Define $A_{*}=P_{\mathscr{A}, *}\left(N\left(x_{*}\right)\right)$ and $B_{*}=C\left(x_{*}\right)+A_{*}$. We assume that $B_{*}$ is nonsingular and

$$
\left|I-B_{*}^{-1} J\left(x_{*}\right)\right| \leq r_{*}<1 .
$$

Assumption DF3. For all $x, z \in \Omega$, there exists $G=G(x, z) \in V(x, z)$ such that

$$
\left\|\left(I-P_{\mathscr{S} \cap Q(0, z-x), *}\right)\left(A_{*}-G\right)\right\|_{*} \leq c_{1} \sigma(x, z)^{p} .
$$

Under Assumptions 1, DF2, and DF3, Dennis and Walker proved the following theorems, which we are also going to prove as particular cases of Theorems 2.1 and 2.5 .

Theorem 3.1. Let $r \in\left(r_{*}, 1\right)$. There exist $\varepsilon=\varepsilon(r)>0$ and $\delta=\delta(r)>0$ such that, if $\left|x_{0}-x_{*}\right| \leq \varepsilon$ and $\left|A_{0}-A_{*}\right| \leq \delta$, the sequence generated by (3.4)-(3.6) is well defined, converges to $x_{*}$, and satisfies

$$
\left|x_{k+1}-x_{*}\right| \leq r\left|x_{k}-x_{*}\right|
$$

for all $k=0,1,2, \ldots$ Moreover, $\left|B_{k}\right|$ and $\left|B_{k}^{-1}\right|$ are uniformly bounded.

Proof. Define $X=\mathbb{R}^{n \times n}, E_{*}=A_{*}$, and $\|\cdot\|_{x z}=\|\cdot\|=\|\cdot\|_{*}$ for all $x, z \in$ $\Omega$. Let $D$ be an open neighborhood of $A_{*}$ such that (perhaps restricting $\Omega$ ) $C(x)+E$ is nonsingular for all $x \in \Omega$ and $E \in D$. Define

$$
\varphi(x, E)=C(x)+E
$$

for all $x \in \Omega$ and $E \in D$.

With the above definitions, the iteration (3.4)-(3.6) has the form (2.2)-(2.4).

Assumption 1 is a hypothesis of Theorem 2.2, and Assumption 2 follows trivially from Assumption DF2. Moreover, Assumption 4 holds trivially in this case. So, in order to prove the theorem, we only need to prove that Assumption 3 follows from Assumption DF3.

For $x, z \in \Omega$, let us define

$$
\widetilde{G}(x, z)=G(x, z)+P_{\mathscr{S} \cap Q(0, z-x)}\left(A_{*}-G(x, z)\right) .
$$

Since $G(x, z) \in V(x, z)$ and $P_{\mathscr{S} \cap Q(0, z-x), *}\left(A_{*}-G\right) \in \mathscr{S} \cap Q(0, z-x)$ (the subspace parallel to $V(x, z))$, we have that $\widetilde{G}(x, z) \in V(x, z)$.

But, by (3.8),

$$
\begin{aligned}
\left\|E_{*}-\tilde{G}(x, z)\right\| & =\left\|A_{*}-G(x, z)-P_{\mathscr{S} \cap Q(0, z-x), *}\left(A_{*}-G(x, z)\right)\right\|_{*} \\
& =\left\|\left(I-P_{\mathscr{S} \cap Q(0, z-x), *}\right)\left(A_{*}-G(x, z)\right)\right\|_{*} \leq c_{1} \sigma(x, z)^{p} .
\end{aligned}
$$


Hence, Assumption 3 holds. Thus, the desired result follows from Theorem 2.1 .

Theorem 3.2. Assume that the sequence generated by (3.4), (3.6), and

$$
B_{k+1}=C\left(x_{k+1}\right)+A_{k+1}
$$

is well defined and that (3.9) holds for all $k=0,1,2, \ldots$. Then

$$
\varlimsup=\frac{\left|x_{k+1}-x_{*}\right|}{\left|x_{k}-x_{*}\right|}=\varlimsup \frac{\left|\left[I-B_{*}^{-1} J\left(x_{*}\right)\right]\left(x_{k}-x_{*}\right)\right|}{\left|x_{k}-x_{*}\right|} \leq r_{*} .
$$

Proof. Define $D=\mathbb{R}^{n \times n}, X, E_{*},\|\cdot\|_{x z},\|\cdot\|$, and $\varphi$ as in the proof of Theorem 3.1. Then, the iteration (3.4), (3.10), (3.6) has the form (2.2), (2.4), (2.5). Since $D=X$, we only need to prove $(2.25)$. Now, for $k=0,1,2, \ldots$,

$$
\begin{aligned}
\left(I-P_{\mathscr{S} \cap Q\left(0, x_{k+1}-x_{k}\right), *}\right)\left(A_{*}-A_{k+1}\right) & \\
= & \left(I-P_{\mathscr{S} \cap Q\left(0, x_{k+1}-x_{k}\right), *}\right)\left(A_{*}-G\left(x_{k}, x_{k+1}\right)\right) \\
& +\left(I-P_{\mathscr{S} \cap Q\left(0, x_{k+1}-x_{k}\right), *}\right)\left(G\left(x_{k}, x_{k+1}\right)-A_{k+1}\right) .
\end{aligned}
$$

But $G\left(x_{k}, x_{k+1}\right)$ and $A_{k+1}$ belong to $V\left(x_{k}, x_{k+1}\right)$. Hence, $G\left(x_{k}, x_{k+1}\right)-$ $A_{k+1} \in \mathscr{S} \cap Q\left(0, x_{k+1}-x_{k}\right)$, and therefore the second term in the right-hand side of (3.12) is null. So, by (3.8), by the convergence of the sequence $\left(x_{k}\right)$, and by the equivalence of norms on $\mathbb{R}^{n \times n}$,

$$
\lim _{k \rightarrow \infty}\left|\left(I-P_{\mathscr{S} \cap Q\left(0, x_{k+1}-x_{k}\right), *}\right)\left(A_{k+1}-A_{*}\right)\right|=0 \text {. }
$$

Now,

$$
\begin{aligned}
& \left|\left(A_{k+1}-A_{*}\right) \frac{\left(x_{k+1}-x_{k}\right)}{\left|x_{k+1}-x_{k}\right|}\right| \\
& \leq\left|\left(I-P_{\mathscr{S} \cap Q\left(0, x_{k+1}-x_{k}\right), *}\right)\left(A_{k+1}-A_{*}\right) \frac{\left(x_{k+1}-x_{k}\right)}{\left|x_{k+1}-x_{k}\right|}\right| \\
& \quad+\left|P_{\mathscr{S} \cap Q\left(0, x_{k+1}-x_{k}\right), *}\left(A_{k+1}-A_{*}\right) \frac{\left(x_{k+1}-x_{k}\right)}{\left|x_{k+1}-x_{k}\right|}\right| .
\end{aligned}
$$

Since $P_{\mathscr{S} \cap Q\left(0, x_{k+1}-x_{k}\right), *}\left(A_{k+1}-A_{*}\right) \in Q\left(0, x_{k+1}-x_{k}\right)$, the second term of the right-hand side of (3.14) is null, and, by (3.13), the first term tends to zero. Therefore,

$$
\lim _{k \rightarrow \infty}\left|\left(A_{k+1}-A_{*}\right) \frac{\left(x_{k+1}-x_{k}\right)}{\left|x_{k+1}-x_{k}\right|}\right|=0
$$

Finally,

$$
\begin{aligned}
& \frac{\left|\left[\varphi\left(x_{k+1}, E_{k+1}\right)-\varphi\left(x_{*}, E_{*}\right)\right]\left(x_{k+1}-x_{k}\right)\right|}{\left|x_{k+1}-x_{k}\right|} \\
& \quad=\frac{\left|\left[\left(C\left(x_{k+1}\right)+A_{k+1}\right)-\left(C\left(x_{*}\right)+A_{*}\right)\right]\left(x_{k+1}-x_{k}\right)\right|}{\left|x_{k+1}-x_{k}\right|} \\
& \quad \leq\left|C\left(x_{k+1}\right)-C\left(x_{*}\right)\right|+\left|\left(A_{k+1}-A_{*}\right) \frac{\left(x_{k+1}-x_{k}\right)}{\left|x_{k+1}-x_{k}\right|}\right| .
\end{aligned}
$$

Hence, (2.25) follows from the convergence of $\left(x_{k}\right)$, the continuity of $C$, and (3.15). This completes the proof. 
Now we are going to describe the "rescaled least-change secant update" method introduced by Dennis and Walker [15].

Assume, as in the case of the fixed-scale least-change secant update method, that $F \in C^{1}(\Omega), J(x)$ has the form (3.1), $C(x)$ is continuous, $\mathscr{A}$ is a linear manifold in $\mathbb{R}^{n \times n}$, and $\mathscr{S}$ is the parallel subspace to $\mathscr{A}$.

Given $x, z \in \Omega$, we choose $v=v(x, z)$ such that $(z-x)^{T} v(x, z)>0$ if $x \neq z$, and a positive definite symmetric matrix $W(x, z)$ such that

$$
W(x, z)(z-x)=v(x, z) .
$$

We define the norm $\|\cdot\|_{x z}$ on $\mathbb{R}^{n \times n}$ by

$$
\|M\|_{x z}=\left[\operatorname{tr}\left\{W(x, z)^{-1} M W(x, z)^{-1} M^{T}\right\}\right]^{1 / 2} .
$$

Given $x, z \in \Omega$, we choose $y=y(x, z) \in \mathbb{R}^{n}$. Remembering the definition (3.2), we define

$$
\begin{aligned}
V(x, z)=\{A \in \mathscr{A} \mid & \operatorname{dist}_{x z}(A, Q(y(x, z), z-x)) \\
& \left.\leq \operatorname{dist}_{x z}\left(A^{\prime}, Q(y(x, z), z-x)\right) \text { for all } A^{\prime} \in \mathscr{A}\right\},
\end{aligned}
$$

where, for any $A \in \mathbb{R}^{n \times n}$ and $\mathscr{C} \subset \mathbb{R}^{n \times n}$,

$$
\operatorname{dist}_{x z}(A, \mathscr{C})=\inf _{B \in \mathscr{C}}\left\{\|A-B\|_{x z}\right\} .
$$

As in the previous case, we know by [13] that $V(x, z)$ is a linear manifold whose parallel subspace is $\mathscr{S} \cap Q(0, z-x)$.

The projection operator onto a set $\mathscr{C} \subset \mathbb{R}^{n \times n}$ with respect to $\|\cdot\|_{x z}$ will be denoted by $P_{\mathscr{C}, x z}$.

The "rescaled least-change secant update" method is defined by the iteration (3.4) $-(3.5)$, where

$$
A_{k+1}=P_{V\left(x_{k}, x_{k+1}\right), x_{k} x_{k+1}}\left(A_{k}\right)
$$

for all $k=0,1,2, \ldots$.

To prove local convergence of (3.4), (3.5), (3.20), suppose that Assumption 1 holds, assume that $J\left(x_{*}\right)$ is positive definite and symmetric, define

$$
\|M\|_{*}=\left[\operatorname{tr}\left\{J\left(x_{*}\right)^{-1} M J\left(x_{*}\right)^{-1} M^{T}\right\}\right]^{1 / 2},
$$

and suppose that Assumption DF2 also holds, where, as always, $P_{\mathscr{C}, *}$ represents the projection with respect to $\|\cdot\|_{*}$. We also need Assumptions DR3 and DR4 below.

Assumption DR3. There exists $\alpha_{1} \geq 0$ such that for all $x, z \in \Omega$, there exists $G=G(x, z) \in V(x, z)$, which satisfies

$$
\left\|\left(I-P_{\mathscr{S} \cap Q(0, z-x), x z}\right)\left(A_{*}-G\right)\right\|_{x z} \leq \alpha_{1} \sigma(x, z)^{p} .
$$

Assumption DR4. (a) For all $x, z \in \Omega$ and $v=v(x, z)$, the projection $P_{\mathscr{S}, x z}(M)$ is independent of the choice of $W(x, z)$ in (3.16). This implies (see [15, p. 972]) that the projection $P_{V(x, z), x z}(M)$ is determined only by $x, z$, and the choice $v(x, z)$ for all $M \in \mathbb{R}^{n \times n}$.

(b) There exists $\alpha_{2} \geq 0$ such that for all $x, z \in \Omega$,

$$
\left|v(x, z)-J\left(x_{*}\right)(z-x)\right| \leq \alpha_{2}|z-x| \sigma(x, z)^{p} .
$$

Under Assumptions 1, DF2, DR3, and DR4, Dennis and Walker [15] proved the following theorems, which we are also going to prove using Theorems 2.12.5. 
Theorem 3.3. Let $r \in\left(r_{*}, 1\right)$. There exist $\varepsilon=\varepsilon(r)>0$ and $\delta=\delta(r)>0$ such that, if $\left|x_{0}-x_{*}\right| \leq \varepsilon$ and $\left|A_{0}-A_{*}\right| \leq \delta$, the sequence generated by (3.4), (3.5), and (3.20) is well defined and satisfies

$$
\left|x_{k+1}-x_{*}\right| \leq r\left|x_{k}-x_{*}\right|
$$

for all $k=0,1,2, \ldots$ Moreover, $\left|B_{k}\right|$ and $\left|B_{k}^{-1}\right|$ are uniformly bounded.

Proof. Define $X=\mathbb{R}^{n \times n}, E_{*}=A_{*}$, and $\|\cdot\|=\|\cdot\|_{*}$. Let $D$ be an open neighborhood of $A_{*}$ such that (perhaps restricting $\Omega$ ) $C(x)+E$ is nonsingular for all $x \in \Omega$ and $E \in D$. Define

$$
\varphi(x, E)=C(x)+E
$$

for all $x \in \Omega$ and $E \in D$.

With the above definitions, the iteration (3.4), (3.5), (3.20) has the form (2.2)-(2.4).

Assumptions 1 and 2 hold trivially as in Theorem 3.1. Let us prove Assumption 4.

Given $x, z \in \Omega, v=v(x, z)$, and $s=z-x$, define

$$
\begin{aligned}
H(x, z)= & J\left(x_{*}\right)+\frac{\left(v-J\left(x_{*}\right) s\right) s^{T}+s\left(v-J\left(x_{*}\right) s\right)^{T}}{s^{T} s} \\
& -\frac{\left(v-J\left(x_{*}\right) s\right)^{T} s s s^{T}}{\left(s^{T} s\right)^{2}} .
\end{aligned}
$$

If $x=z$, we define $H(x, z)=J\left(x_{*}\right) . \quad(H(x, z)$ is the closest symmetric matrix to $J\left(x_{*}\right)$ which belongs to $Q(v, s)$ in the Frobenius norm. See [14, $\mathrm{p}$. $196 ; 31]$.

By (3.23) and (3.25), we have

$$
\left|J\left(x_{*}\right)-H(x, z)\right| \leq 3 \alpha_{2} \sigma(x, z)^{p} .
$$

Therefore, there exists $\Omega_{1} \subset \Omega, \Omega_{1}$ an open neighborhood of $x_{*}$, such that $H(x, z)$ is positive definite for all $x, z \in \Omega_{1}$. For simplicity we also denote $\Omega_{1}$ by $\Omega$.

Now, by Assumption DR4(a), the projection $P_{V(x, z), x z}$ is independent of the choice of $W(x, z)$ in (3.16). In particular, since $H(x, z)(z-x)=v(x, z)$, we may consider that $H(x, z)$ is the weight matrix which defines the projections, that is, we may define

$$
\|M\|_{x z}=\left[\operatorname{tr}\left\{H(x, z)^{-1} M H(x, z)^{-1} M^{T}\right\}\right]^{1 / 2}
$$

for all $M \in \mathbb{R}^{n \times n}$.

Assume that $L(x, z)$ and $L_{*}$ are lower triangular matrices with positive diagonal elements (Cholesky factors) such that for all $x, z \in \Omega$,

$$
L(x, z) L(x, z)^{T}=H(x, z)
$$

and $L_{*} L_{*}^{T}=J\left(x_{*}\right)$. Then, for all $x, z \in \Omega$,

$$
\|M\|_{x z}=\left\|L(x, z)^{-1} M L(x, z)^{-T}\right\|_{F}
$$

and

$$
\|M\|=\left\|L_{*}^{-1} M L_{*}^{-T}\right\|_{F} .
$$


The relations (3.26), (3.27), and (3.28) imply that for all $x, z \in \Omega$,

$$
\left\|L(x, z)-L_{*}\right\| \leq K \sigma(x, z)^{p}
$$

and

$$
\left\|L(x, z)^{-1}-L_{*}^{-1}\right\| \leq K \sigma(x, z)^{p}
$$

for a suitably defined $K>0$. (See [28, proof of Theorem 4.3].) Moreover, (3.27), (3.28), and (3.30) lead to (2.10) and (2.11), using straightforward algebraic manipulations. Thus, Assumption 4 is proved.

Let us now prove Assumption 3. For $x, z \in \Omega$, define

$$
\widetilde{G}(x, z)=G(x, z)+P_{\mathscr{S} \cap Q(0, z-x), x z}\left(A_{*}-G(x, z)\right) .
$$

Since $G(x, z) \in V(x, z)$ and $P_{\mathscr{S} \cap Q(0, z-x), x z}\left(A_{*}-G(x, z)\right) \in \mathscr{S} \cap Q(0, z-x)$, we have that $\widetilde{G}(x, z) \in V(x, z)$.

But, by (3.22),

$$
\begin{aligned}
\left\|E_{*}-\tilde{G}(x, z)\right\|_{x z} & =\left\|A_{*}-G(x, z)-P_{\mathscr{S} \cap Q(0, z-x), x z}\left(A_{*}-G(x, z)\right)\right\|_{x z} \\
& =\left\|\left(I-P_{\mathscr{S} \cap Q(0, z-x), x z}\right)\left(A_{*}-G(x, z)\right)\right\|_{x z} \leq \alpha_{1} \sigma(x, z)^{p} .
\end{aligned}
$$

Therefore, by the already proved Assumption 4,

$$
\begin{aligned}
\left\|E_{*}-\tilde{G}(x, z)\right\| & \leq\left\|E_{*}-\widetilde{G}(x, z)\right\|_{x z}\left(1+c_{2} \sigma(x, z)^{p}\right) \\
& \leq \alpha_{1} \sigma(x, z)^{p}\left(1+c_{2} \sigma(x, z)^{p}\right) .
\end{aligned}
$$

Hence, Assumption 3 follows trivially. Thus, the desired result follows from Theorem 2.1.

Theorem 3.4. Assume that the sequence generated by (3.4), (3.20), and

$$
B_{k+1}=C\left(x_{k+1}\right)+A_{k+1}
$$

is well defined and that (3.24) holds for all $k=0,1,2, \ldots$ Then

$$
\varlimsup \frac{\left|x_{k+1}-x_{*}\right|}{\left|x_{k}-x_{*}\right|}=\varlimsup \frac{\left|\left[I-B_{*}^{-1} J\left(x_{*}\right)\right]\left(x_{k}-x_{*}\right)\right|}{\left|x_{k}-x_{*}\right|} \leq r_{*} .
$$

Proof. Define $D=\mathbb{R}^{n \times n}, X, E_{*},\|\cdot\|_{x z},\|\cdot\|$, and $\varphi$ as in the proof of Theorem 3.3. Since $D=X$, we only need to prove (2.25).

Since both $G\left(x_{k}, x_{k+1}\right)$ and $A_{k+1}$ belong to $V\left(x_{k}, x_{k+1}\right)$, we have that $G\left(x_{k}, x_{k+1}\right)-A_{k+1} \in \mathscr{S} \cap Q\left(0, x_{k+1}-x_{k}\right)$. Therefore, by (3.22) and the convergence of $\left(x_{k}\right)$,

$$
\begin{aligned}
& \left\|\left(I-P_{\mathscr{S} \cap Q\left(0, x_{k+1}-x_{k}\right), x_{k} x_{k+1}}\right)\left(A_{*}-A_{k+1}\right)\right\|_{x_{k} x_{k+1}}
\end{aligned}
$$

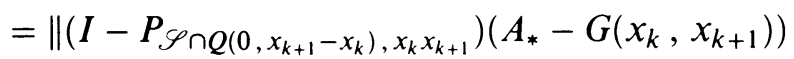

$$
\begin{aligned}
& +\left(I-P_{\mathscr{S} \cap Q\left(0, x_{k+1}-x_{k}\right), x_{k} x_{k+1}}\right)\left(G\left(x_{k}, x_{k+1}\right)-A_{k+1}\right) \|_{x_{k} x_{k+1}} \\
& =\left\|\left(I-P_{\mathscr{P} \cap Q\left(0, x_{k+1}-x_{k}\right), x_{k} x_{k+1}}\right)\left(A_{*}-G\left(x_{k}, x_{k+1}\right)\right)\right\|_{x_{k} x_{k+1}} \rightarrow 0 .
\end{aligned}
$$
$\mathbb{R}^{n \times n}$

$$
\lim _{k \rightarrow \infty}\left|\left(I-P_{\mathscr{P} \cap Q\left(0, x_{k+1}-x_{k}\right), x_{k} x_{k+1}}\right)\left(A_{k+1}-A_{*}\right)\right|=0 .
$$

Finally, (3.32) follows from (3.33) exactly in the way as it was deduced from (3.13) in the proof of Theorem 3.2. 


\section{LOCAL CONVERGENCE OF THE INVERSE LEAST-CHANGE SECANT UPDATE METHODS}

The inverse least-change secant update methods were also introduced by Dennis and Walker [15]. Unlike the direct least-change secant update methods, which, at each iteration, update an approximation of a part of the Jacobian matrix, the inverse least-change secant update methods update, at each iteration, a part of the inverse of the Jacobian.

We begin with describing the fixed-scale inverse least-change secant update method.

As in (2.1), assume that $F \in C^{1}(\Omega), \Omega$ an open and convex set of $\mathbb{R}^{n}$, and that, for all $x \in \Omega$,

$$
J(x)^{-1}=C(x)+N(x),
$$

where $C(x)$ is continuous in $\Omega$.

Let $\mathscr{A} \subset \mathbb{R}^{n \times n}$ be a linear manifold, and denote by $\mathscr{S}$ the parallel subspace to $\mathscr{A}$.

Let $\|\cdot\|_{*}$ be a norm on $\mathbb{R}^{n \times n}$, associated with some scalar product. The projection operator onto a set $\mathscr{C} \subset \mathbb{R}^{n \times n}$ with respect to $\|\cdot\|_{*}$ will be denoted by $P_{\mathscr{C}, *}$.

For all $x, z \in \Omega$, we choose $w=w(x, z) \in \mathbb{R}^{n}$ and $y=y(x, z) \in \mathbb{R}^{n}$, with $y(x, z) \neq 0$ if $x \neq z$. We also define

$$
\begin{aligned}
V(x, z)=\{A \in \mathscr{A} \mid & \operatorname{dist}_{*}(A, Q(w(x, z), y(x, z))) \\
& \left.\leq \operatorname{dist}_{*}\left(A^{\prime}, Q(w(x, z), y(x, z))\right) \text { for all } A^{\prime} \in \mathscr{A}\right\},
\end{aligned}
$$

where $Q$ and dist $_{*}$ are defined as in (3.2) and (3.3), respectively.

As in $\S 3$, we know from [13] that $V(x, z)$ is a linear manifold and that the parallel subspace to $V(x, z)$ is $\mathscr{S} \cap Q(0, y(x, z))$.

The inverse fixed-scale least-change secant update method proposed by Dennis and Walker [15] consists of the iteration

$$
x_{k+1}=x_{k}-K_{k} F\left(x_{k}\right),
$$

where

$$
K_{k+1} \in\left\{C\left(x_{k}\right)+A_{k}, C\left(x_{k+1}\right)+A_{k}, C\left(x_{k}\right)+A_{k+1}, C\left(x_{k+1}\right)+A_{k+1}\right\}
$$

and

$$
A_{k+1}=P_{V\left(x_{k}, x_{k+1}\right), *} .
$$

The first assumption which we need to prove that the sequence generated by (4.3), (4.4), and (4.5) is well defined and converges to a solution of $F(x)=0$ is Assumption 1 of $\S 2$. We also need assumptions IF2 and IF3 below.

Assumption IF2. Define $A_{*}=P_{\mathscr{A}, *}\left(N\left(x_{*}\right)\right)$ and $K_{*}=C\left(x_{*}\right)+A_{*}$. There exists $r_{*} \in[0,1)$ such that

$$
\left|I-K_{*} J\left(x_{*}\right)\right| \leq r_{*} .
$$

Since $J\left(x_{*}\right)$ is nonsingular by Assumption 1, (4.6) implies that $K_{*}$ is nonsingular. 
Assumption IF3. For all $x, z \in \Omega$, there exists $G=G(x, z) \in V(x, z)$ such that

$$
\left\|\left(I-P_{\mathscr{S} \cap Q(0, y(x, z))}\right)\left(A_{*}-G\right)\right\|_{*} \leq c_{1} \sigma(x, z)^{p}
$$

Using Assumptions 1, IF2, and IF3, Dennis and Walker proved the following theorems, which we are also going to prove using Theorems 2.1-2.5.

Theorem 4.1. Let $r \in\left(r_{*}, 1\right)$. There exist $\varepsilon=\varepsilon(r)$ and $\delta=\delta(r)$ such that, if $\left|x_{0}-x_{*}\right| \leq \varepsilon$ and $\left|A_{0}-A_{*}\right| \leq \delta$, the sequence generated by (4.3), (4.4), and (4.5) is well defined, converges to $x_{*}$, and satisfies

$$
\left|x_{k+1}-x_{*}\right| \leq r\left|x_{k}-x_{*}\right|
$$

for all $k=0,1,2, \ldots$. Moreover, $\left|K_{k}\right|$ and $\left|K_{k}^{-1}\right|$ are uniformly bounded.

Proof. Define $X=\mathbb{R}^{n \times n}, E_{*}=A_{*}$, and $\|\cdot\|_{x z}=\|\cdot\|=\|\cdot\|_{*}$ for all $x, z \in$ $\Omega$. Let $D$ be an open neighborhood of $A_{*}$ such that (perhaps restricting $\Omega$ ) $C(x)+E$ is nonsingular for all $x \in \Omega$ and $E \in D$. Define

$$
\varphi(x, E)=(C(x)+E)^{-1}
$$

for all $x \in \Omega$ and $E \in D$.

With these definitions, the algorithm (4.3)-(4.5) has the form (2.2)-(2.4).

Assumption 1 is a hypothesis of this theorem, and Assumption 2 follows trivially from Assumption IF2. Clearly, Assumption 4 is also trivially satisfied. So, let us prove that Assumption 3 may be deduced from Assumption IF3.

For all $x, z \in \Omega$, we define

$$
\widetilde{G}(x, z)=G(x, z)+P_{\mathscr{S} \cap Q(0, y(x, z)), *}\left(A_{*}-G(x, z)\right) .
$$

Since $G(x, z) \in V(x, z)$ and $P_{\mathscr{S} \cap Q(0, y(x, z)), *}\left(A_{*}-G(x, z)\right)$ belongs to the parallel subspace to $V(x, z)$, we have that $\widetilde{G}(x, z) \in V(x, z)$. Now, by (4.7),

$$
\begin{aligned}
\left\|E_{*}-\tilde{G}(x, z)\right\| & =\left\|A_{*}-G(x, z)-P_{\mathscr{S} \cap Q(0, y(x, z)), *}\left(A_{*}-G(x, z)\right)\right\|_{*} \\
& \leq\left\|\left(I-P_{\mathscr{S} \cap Q(0, y(x, z)), *}\right)\left(A_{*}-G(x, z)\right)\right\|_{*} \leq c_{1} \sigma(x, z)^{p} .
\end{aligned}
$$

So, Assumption 3 is proved. Hence, the desired result follows from Theorem 2.1 .

Theorem 4.2. Assume that the sequence generated by (4.3), (4.5), and

$$
K_{k+1}=C\left(x_{k+1}\right)+A_{k+1}
$$

is well defined and that (4.8) holds for all $k=0,1,2, \ldots$ Additionally, assume that

$$
\lim _{k \rightarrow \infty} \frac{\left|K_{*} y\left(x_{k}, x_{k+1}\right)-\left(x_{k+1}-x_{k}\right)\right|}{\left|x_{k+1}-x_{k}\right|}=0
$$

and that $\left|K_{k}\right|$ and $\left|K_{k}^{-1}\right|$ are uniformly bounded. Then

$$
\varlimsup \frac{\left|x_{k+1}-x_{*}\right|}{\left|x_{k}-x_{*}\right|}=\varlimsup \frac{\left|\left(I-\left[C\left(x_{*}\right)+A_{*}\right] J\left(x_{*}\right)\right)\left(x_{k}-x_{*}\right)\right|}{\left|x_{k}-x_{*}\right|} \leq r_{*} .
$$


Proof. Let $M>0$ be such that $\left|K_{k}\right| \leq M$ and $\left|K_{k}^{-1}\right| \leq M$ for all $k=$ $0,1,2, \ldots$. Using the definitions given in the proof of Theorem 4.1, we have that, for all $k=0,1,2, \ldots$,

$$
\begin{array}{r}
\left(x_{k}, E_{k}\right) \in \Gamma=\left\{(x, E) \in \mathbb{R}^{n} \times X|| \varphi(x, E)|\leq M,| \varphi(x, E)^{-1} \mid \leq M\right. \\
\text { and } \left.\left|x-x_{*}\right| \leq\left|x_{0}-x_{*}\right|\right\} .
\end{array}
$$

Clearly, $\Gamma$ is a closed set. So, by Theorem 2.5 , we only need to prove (2.25).

Now, for $k=0,1,2, \ldots$,

$$
\begin{aligned}
\left(I-P_{\mathscr{S} \cap Q\left(0, y\left(x_{k}, x_{k+1}\right)\right), *}\right)\left(A_{*}-A_{k+1}\right) \\
=\left(I-P_{\mathscr{S} \cap Q\left(0, y\left(x_{k}, x_{k+1}\right)\right), *}\right)\left(A_{*}-G\left(x_{k}, x_{k+1}\right)\right) \\
\quad+\left(I-P_{\mathscr{S} \cap Q\left(0, y\left(x_{k}, x_{k+1}\right)\right), *}\right)\left(G\left(x_{k}, x_{k+1}\right)-A_{k+1}\right) .
\end{aligned}
$$

Since $G\left(x_{k}, x_{k+1}\right)-A_{k+1} \in \mathscr{S} \cap Q\left(0, y\left(x_{k}, x_{k+1}\right)\right)$, the second term of the right-hand side of $(4.13)$ is null for all $k=0,1,2, \ldots$ So, by (4.7), the convergence of $\left(x_{k}\right)$, and the equivalence of norms in $\mathbb{R}^{n \times n}$, we have

$$
\lim _{k \rightarrow \infty}\left|\left(I-P_{\mathscr{S} \cap Q\left(0, y\left(x_{k}, x_{k+1}\right)\right), *}\right)\left(A_{*}-A_{k+1}\right)\right|=0 .
$$

By (4.11), for large enough $k$, we have $y\left(x_{k}, x_{k+1}\right) \neq 0$. Therefore, by (4.14),

$$
\begin{aligned}
\mid\left(A_{k+1}\right. & \left.-A_{*}\right) \frac{y\left(x_{k}, x_{k+1}\right)}{\left|y\left(x_{k}, x_{k+1}\right)\right|} \mid \\
\leq & \left|\left(I-P_{\mathscr{S} \cap Q\left(0, y\left(x_{k}, x_{k+1}\right)\right), *}\right)\left(A_{k+1}-A_{*}\right) \frac{y\left(x_{k}, x_{k+1}\right)}{\left|y\left(x_{k}, x_{k+1}\right)\right|}\right| \\
& +\left|P_{\mathscr{S} \cap Q\left(0, y\left(x_{k}, x_{k+1}\right)\right), *}\left(A_{k+1}-A_{*}\right) \frac{y\left(x_{k}, x_{k+1}\right)}{\left|y\left(x_{k}, x_{k+1}\right)\right|}\right| \\
= & \left|\left(I-P_{\mathscr{S} \cap Q\left(0, y\left(x_{k}, x_{k+1}\right)\right), *}\right)\left(A_{k+1}-A_{*}\right) \frac{y\left(x_{k}, x_{k+1}\right)}{\left|y\left(x_{k}, x_{k+1}\right)\right|}\right| \\
\leq & \left|\left(I-P_{\mathscr{S} \cap Q\left(0, y\left(x_{k}, x_{k+1}\right)\right), *}\right)\left(A_{k+1}-A_{*}\right)\right| \rightarrow 0 \text { as } k \rightarrow \infty .
\end{aligned}
$$

Now, by the uniform boundedness of $\left|\varphi\left(x_{k}, E_{k}\right)^{-1}\right|$,

$$
\begin{aligned}
& \frac{\left|\left[\left(C\left(x_{k+1}\right)+A_{k+1}\right)^{-1}-\left(C\left(x_{*}\right)+A_{*}\right)^{-1}\right] K_{*} y\left(x_{k}, x_{k+1}\right)\right|}{\left|K_{*} y\left(x_{k}, x_{k+1}\right)\right|} \\
& \quad \leq \frac{\left|\left[C\left(x_{k+1}\right)+A_{k+1}\right]^{-1}\right|\left|\left[\left(C\left(x_{k+1}\right)+A_{k+1}\right)-\left(C\left(x_{*}\right)+A_{*}\right)\right] y\left(x_{k}, x_{k+1}\right)\right|}{\left|K_{*} y\left(x_{k}, x_{k+1}\right)\right|} \\
& \quad \leq \beta_{1} \frac{\left|\left[\left(C\left(x_{k+1}\right)+A_{k+1}\right)-\left(C\left(x_{*}\right)+A_{*}\right)\right] y\left(x_{k}, x_{k+1}\right)\right|}{\left|K_{*} y\left(x_{k}, x_{k+1}\right)\right|}
\end{aligned}
$$

for a suitably defined constant $\beta_{1}>0$.

Therefore, by (4.19), (4.16), and the continuity of $C$, we have

$$
\lim _{k \rightarrow \infty} \frac{\left|\left[\varphi\left(x_{k+1}, E_{k+1}\right)-\varphi\left(x_{*}, E_{*}\right)\right] K_{*} y\left(x_{k}, x_{k+1}\right)\right|}{\left|K_{*} y\left(x_{k}, x_{k+1}\right)\right|}=0 .
$$


Hence, by (4.11), (4.17), and the uniform boundedness of $\left|\varphi\left(x_{k}, E_{k}\right)\right|$,

$$
\begin{aligned}
\lim _{k \rightarrow \infty} & \frac{\left|\left[\varphi\left(x_{k+1}, E_{k+1}\right)-\varphi\left(x_{*}, E_{*}\right)\right]\left(x_{k+1}-x_{k}\right)\right|}{\left|x_{k+1}-x_{k}\right|} \\
& \leq \lim _{k \rightarrow \infty}\left\{\frac{\left|\left[\varphi\left(x_{k+1}, E_{k+1}\right)-\varphi\left(x_{*}, E_{*}\right)\right]\left[\left(x_{k+1}-x_{k}\right)-K_{*} y\left(x_{k}, x_{k+1}\right)\right]\right|}{\left|K_{*} y\left(x_{k}, x_{k+1}\right)\right|}\right. \\
\left.+\frac{\left|\left[\varphi\left(x_{k+1}, E_{k+1}\right)-\varphi\left(x_{*}, E_{*}\right)\right] K_{*} y\left(x_{k}, x_{k+1}\right)\right|}{\left|K_{*} y\left(x_{k}, x_{k+1}\right)\right|}\right\} & \cdot \frac{\left|x_{k+1}-x_{k}\right|}{\left|K_{*} y\left(x_{k}, x_{k+1}\right)\right|} \\
& \leq \lim _{k \rightarrow \infty} \frac{\left|\left[\varphi\left(x_{k+1}, E_{k+1}\right)-\varphi\left(x_{*}, E_{*}\right)\right]\left[\left(x_{k+1}-x_{k}\right)-K_{*} y\left(x_{k}, x_{k+1}\right)\right]\right|}{\left|x_{k+1}-x_{k}\right|} \\
& \leq \lim _{k \rightarrow \infty} \mid\left[\varphi\left(x_{k+1}, E_{k+1}\right)-\varphi\left(x_{*}, E_{*}\right)\right] \frac{\left[\left(x_{k+1}-x_{k}\right)-K_{*} y\left(x_{k}, x_{k+1}\right)\right]}{\left|x_{k+1}-x_{k}\right|} \cdot \frac{\left|x_{k+1}-x_{k}\right|}{\left|K_{*} y\left(x_{k}, x_{k+1}\right)\right|} \\
& =0 .
\end{aligned}
$$

This completes the proof of (4.12).

We finish this section proving convergence of the "inverse rescaled leastchange secant update" method of Dennis and Walker.

We assume again that $F \in C^{1}(\Omega), J(x)^{-1}$ has the form (4.1), $C$ is continuous, $\mathscr{A}$ is a linear manifold, and $\mathscr{S}$ is the parallel subspace to $\mathscr{A}$.

Given $x, z \in \Omega$, we choose $u(x, z), w(x, z), y(x, z) \in \mathbb{R}^{n}$ such that $y(x, z) \neq 0$ and $u(x, z)^{T} y(x, z)>0$ if $x \neq z$. We also choose a positive definite symmetric matrix $W(x, z) \in \mathbb{R}^{n \times n}$ such that

$$
W(x, z) y(x, z)=u(x, z) .
$$

We define the norm $\|\cdot\|_{x z}$ on $\mathbb{R}^{n \times n}$ by

$$
\|M\|_{x z}=\left[\operatorname{tr}\left\{W(x, z)^{-1} M W(x, z)^{-1} M^{T}\right\}\right]^{1 / 2}
$$

as in (3.17), and

$$
\begin{aligned}
V(x, z)=\{A \in \mathscr{A} \mid & \operatorname{dist}_{x z}(A, Q(w(x, z), y(x, z))) \\
& \left.\leq \operatorname{dist}_{x z}\left(A^{\prime}, Q(w(x, z), y(x, z))\right) \text { for all } A^{\prime} \in \mathscr{A}\right\} .
\end{aligned}
$$

As before, remember that Dennis and Schnabel [13] proved that $V(x, z)$ is a linear manifold whose parallel subspace is $\mathscr{S} \cap Q(0, y(x, z))$. Denote by $P_{\mathscr{C}, x z}$ the projection operator onto a set $\mathscr{C} \subset \mathbb{R}^{n \times n}$ with respect to the norm $\|\cdot\|_{x z}$.

The "inverse rescaled least-change secant update" method is defined by the iteration (4.3)-(4.4), where

$$
A_{k+1}=P_{V\left(x_{k}, x_{k+1}\right), x_{k} x_{k+1}}\left(A_{k}\right)
$$

for all $k=0,1,2, \ldots$.

Suppose that Assumption 1 of $\S 2$ holds, assume that $J\left(x_{*}\right)$ is positive definite and symmetric, and define

$$
\|M\|_{*}=\left[\operatorname{tr}\left\{J\left(x_{*}\right)^{-1} M J\left(x_{*}\right)^{-1} M^{T}\right\}\right]^{1 / 2}
$$


for all $M \in \mathbb{R}^{n \times n}$. Suppose, further, that Assumption IF2 also holds. Together with Assumptions 1 and IF2, the following Assumptions IR3 and IR4 are necessary to prove that the algorithm is well defined and locally convergent.

Assumption IR3. There exists $\alpha_{1} \geq 0$ such that for all $x, z \in \Omega$, there exists $G=G(x, z) \in V(x, z)$ satisfying

$$
\left\|\left(I-P_{\mathscr{S} \cap Q(0, y(x, z)), x z}\right)\left(A_{*}-G\right)\right\|_{x z} \leq \alpha_{1} \sigma(x, z)^{p} .
$$

Assumption IR4. (a) For all $x, z \in \Omega, u=u(x, z)$, and $y=y(x, z)$, the projection $P_{\mathscr{S}, x z}(M)$ is independent of the choice of $W(x, z)$ in (4.18). Then, by [15, p. 972], the projection $P_{V(x, z), x z}(M)$ is determined only by $x, z$, and the choices of $u(x, z)$ and $y(x, z)$.

(b) There exists $\alpha_{2} \geq 0$ such that for all $x, z \in \Omega$,

$$
\left|u(x, z)-J\left(x_{*}\right)^{-1} y(x, z)\right| \leq \alpha_{1}|y(x, z)| \sigma(x, z)^{p} .
$$

Let us prove now a local convergence theorem for the algorithm defined by (4.3), (4.4), and (4.20). We will use Assumptions 1, IF2, IR3, and IR4 for placing this algorithm under the hypotheses of Theorem 2.1.

Theorem 4.3. Let $r \in\left(r^{*}, 1\right)$. There exist $\varepsilon=\varepsilon(r)$ and $\delta=\delta(r)$ such that, if $\left|x_{0}-x_{*}\right| \leq \varepsilon$ and $\left|A_{0}-A_{*}\right| \leq \delta$, the sequence generated by (4.3), (4.4), and (4.20) is well defined, converges to $x_{*}$, and satisfies (4.8) for all $k=0,1,2, \ldots$ Moreover, $\left|K_{k}\right|$ and $\left|K_{k}^{-1}\right|$ are uniformly bounded.

Proof. Define $X=\mathbb{R}^{n \times n}, E_{*}=A_{*}$, and $\|\cdot\|=\|\cdot\|_{*}$. Let $D$ be an open neighborhood of $A_{*}$ such that (perhaps restricting $\Omega$ ) $C(x)+E$ is nonsingular for all $x \in \Omega$ and $E \in D$. Define

$$
\varphi(x, E)=(C(x)+E)^{-1}
$$

for all $x \in \Omega$ and $E \in D$.

Now, the algorithm (4.3), (4.4), (4.20) has the form (2.2)-(2.4). Since Assumption 1 is assumed to hold, and Assumption 2 follows from Assumption IF2, we need to prove Assumptions 3 and 4. Let us prove Assumption 4.

Given $x, z \in \Omega, x \neq z, y=y(x, z)$, and $u=u(x, z)$, we define

$$
\begin{aligned}
H(x, z)= & J\left(x_{*}\right)^{-1}+\frac{\left(u-J\left(x_{*}\right)^{-1} y\right) y^{T}+y\left(u-J\left(x_{*}\right)^{-1} y\right)^{T}}{y^{T} y} \\
& -\frac{\left(u-J\left(x_{*}\right)^{-1} y\right)^{T} y y y^{T}}{\left(y^{T} y\right)^{2}} .
\end{aligned}
$$

If $x=z$, we define $H(x, z)=J\left(x_{*}\right)^{-1}$.

Since $y(x, z) \neq 0, H(x, z)$ is well defined for all $x, z \in \Omega, x \neq z$. $\left(H(x, z)\right.$ is the closest symmetric matrix to $J\left(x_{k}\right)^{-1}$ in the Frobenius norm which satisfies $H y=u$.) By (4.22) and (4.23), we have that

$$
\left|H(x, z)-J\left(x_{*}\right)^{-1}\right| \leq 3 \alpha_{2} \sigma(x, z)^{p} .
$$

Therefore, $H(x, z)$ is positive definite if $x, z \in \Omega_{1} \subset \Omega, \Omega_{1}$ an open and convex set. For simplicity we also denote $\Omega_{1}$ by $\Omega$. By Assumption IR4(a) we may consider that $W(x, z)=H(x, z)$ in (4.18). Finally, Assumption 4 follows using the arguments used in Theorem 3.3. 
Let us now prove Assumption 3. For $x, z \in \Omega$, define

$$
\widetilde{G}(x, z)=G(x, z)+P_{\mathscr{S} \cap Q(0, y(x, z)), x z}\left(A_{*}-G(x, z)\right) .
$$

Since $G(x, z)$ belongs to $V(x, z)$, we have that $\widetilde{G}(x, z)$ belongs to $V(x, z)$ and $P_{\mathscr{S} \cap Q(0, y(x, z)), x z}\left(A_{*}-G(x, z)\right)$ belongs to the parallel subspace to $V(x, z)$.

Clearly, by (4.21),

$$
\begin{aligned}
\| E_{*}- & \tilde{G}(x, z) \|_{x z} \\
& =\left\|A_{*}-G(x, z)-P_{\mathscr{S} \cap Q(0, y(x, z)), x z}\left(A_{*}-G(x, z)\right)\right\|_{x z} \\
& =\left\|\left(I-P_{\mathscr{S} \cap Q(0, y(x, z)), x z}\right)\left(A_{*}-G(x, z)\right)\right\|_{x z} \leq \alpha_{1} \sigma(x, z)^{p} .
\end{aligned}
$$

Therefore, Assumption 3 follows from (4.25) and from Assumption 4. Hence, the desired result follows from Theorem 2.1.

Theorem 4.4. Assume that the sequence generated by (4.3), (4.20), and

$$
K_{k+1}=C\left(x_{k+1}\right)+A_{k+1}
$$

is well defined and that (4.8) holds for all $k=0,1,2, \ldots$. Additionally, assume that

$$
\lim _{k \rightarrow \infty} \frac{\left|K_{*} y\left(x_{k}, x_{k+1}\right)-\left(x_{k+1}-x_{k}\right)\right|}{\left|x_{k+1}-x_{k}\right|}=0
$$

and that $\left|K_{k}\right|$ and $\left|K_{k}^{-1}\right|$ are uniformly bounded. Then

$$
\varlimsup \frac{\left|x_{k+1}-x_{*}\right|}{\left|x_{k}-x_{*}\right|}=\varlimsup \frac{\left|\left(I-\left[C\left(x_{*}\right)+A_{*}\right] J\left(x_{*}\right)\right)\left(x_{k}-x_{*}\right)\right|}{\left|x_{k}-x_{*}\right|} \leq r_{*} .
$$

Proof. The desired result is obtained repeating the proof of Theorem 4.2.

\section{GENERALIZATION OF THE SECANT-FINITE DIFFERENCE METHOD}

The Secant-Finite Difference (SFD) method was introduced by Dennis and Li [11]. The objective of this method is to save function evaluations required by the discrete Newton method by using the previous iteration properly.

Assume that the set of column indices $I_{n}=\{1, \ldots, n\}$ is partitioned into $q$ nonvoid disjoint subsets $\pi_{1}, \ldots, \pi_{q}$. Without loss of generality, assume that $\pi_{1}=\left\{1, \ldots, n_{1}\right\}, \pi_{2}=\left\{n_{1}+1, \ldots, n_{1}+n_{2}\right\}, \ldots, \pi_{q}=\left\{\sum_{j=1}^{q-1} n_{j}+1, \ldots, n\right\}$. Dennis and $\mathrm{Li}$ [11] suggest partitioning $I_{n}$ using the CPR property [7] together with the algorithm of Coleman and More [6]. The integer $q$ is chosen so that $\left\{\pi_{2}, \ldots, \pi_{q}\right\}$ is a CPR-partition and $\pi_{1}$ contains the remaining columns. However, our analysis permits a completely arbitrary partition. Given any matrix $B \in \mathbb{R}^{n \times n}$, let us write $B=\left(B^{1}, \ldots, B^{q}\right)$, where $B^{j}$ is an $n \times n_{j}$ matrix which contains the columns corresponding to the indices of $\pi_{j}$.

Consider a function $F: \Omega \subset \mathbb{R}^{n} \rightarrow \mathbb{R}^{n}, F \in C^{1}(\Omega), \Omega$ an open Euclidean ball. For all $x \in \mathbb{R}^{n}$, write

$$
x=\left(\begin{array}{c}
x^{1} \\
\vdots \\
x^{q}
\end{array}\right),
$$

where $x^{j}=\left(e_{m_{j}+1}^{T} x, \ldots, e_{m_{j}+n_{j}}^{T} x\right)^{T}, m_{j}=\sum_{l=1}^{j-1} n_{l}, j=2, \ldots, q, m_{1}=0$. 
For all $x \in \Omega$, we assume that $J(x)=\left(J_{1}(x), \ldots, J_{q}(x)\right)$ is such that $J_{j}(x) \in \mathscr{S}_{j} \subset \mathbb{R}^{n \times n_{j}}$ for $j=1, \ldots, q$, where $\mathscr{S}_{j}$ is a linear manifold.

Assume that $x_{0} \in \Omega$ and $B_{0}$ is a nonsingular $n \times n$ matrix. The $k$ th iteration of the Generalized Secant-Finite Difference (GSFD) method is defined by the following algorithm.

\section{Algorithm 5.1.}

Step 1. Given $x_{k} \in \Omega$ and $B_{k}=\left(B_{k}^{1}, \ldots, B_{k}^{q}\right)$, solve

$$
B_{k} s_{k}=-F\left(x_{k}\right), \quad s_{k}=\left(\begin{array}{c}
s_{k}^{1} \\
\vdots \\
s_{k}^{q}
\end{array}\right),
$$

and set

$$
x_{k+1}=x_{k}+s_{k} .
$$

Step 2. For $j=1, \ldots, q$, solve, for $B_{k+1}^{j}$,

$$
\begin{aligned}
& \text { Minimize }\left\|B-B_{k}^{j}\right\|_{F} \\
& \text { s.t. } B s_{k}^{j}=F\left(\tilde{x}_{k}^{j}\right)-F\left(\tilde{x}_{k}^{j-1}\right), \\
& B \in \mathscr{S}_{j},
\end{aligned}
$$

where

$$
\tilde{x}_{k}^{j}=\left(\begin{array}{c}
x_{k+1}^{1} \\
\vdots \\
x_{k+1}^{j} \\
x_{k}^{j+1} \\
\vdots \\
x_{k}^{q}
\end{array}\right) \quad \text { for } j=1, \ldots, q .
$$

(So, $\tilde{x}_{k}^{0}=x_{k}$ and $\tilde{x}_{k}^{q}=x_{k+1}$.)

Step 3. $B_{k+1}=\left(B_{k+1}^{1}, \ldots, B_{k+1}^{q}\right)$.

If $q=n$, GSFD is the method described in [30, pp. 196-197, formula (21)]. Except for the choice of the partition, Algorithm 5.1 is identical to the SFD algorithm of Dennis and Li. Those authors gave a convergence analysis for SFD, which does not rely on the Dennis-Walker theory. In what follows, we prove local and superlinear convergence of GSFD, under the usual assumptions made in these cases by using Martínez's theory in an almost straightforward way.

First, let us formulate GSFD in such a way that it becomes evident that it is a particular case of Algorithm 2.2-2.5.

Define $X=\mathbb{R}^{n \times n_{1}} \times \cdots \times \mathbb{R}^{n \times n_{q}}$ (of course, there exists an obvious isomorphism between $X$ and $\mathbb{R}^{n \times n}$, but we feel that the formulation in terms of $X$ is more clear), $\left\|\left(B^{1}, \ldots, B^{q}\right)\right\|=\left(\sum_{j=1}^{q}\left\|B^{j}\right\|_{F}^{2}\right)^{1 / 2},\|\cdot\|_{x z}=\|\cdot\|$ for all $x, z \in$ $\Omega$, and $\varphi\left(x,\left(B^{1}, \ldots, B^{q}\right)\right)=\left(B^{1}, \ldots, B^{q}\right)$ for all $x \in \Omega,\left(B^{1}, \ldots, B^{q}\right) \in X$. 
Given $x, z \in \Omega$, we define the vectors $\xi_{j}(x, z), j=0,1, \ldots, q$, by

$$
\xi_{j}(x, z)=\left(\begin{array}{c}
z^{1} \\
\vdots \\
z^{j} \\
x^{j+1} \\
\vdots \\
x^{q}
\end{array}\right) .
$$

So, $\xi_{0}(x, z)=x$ and $\xi_{q}(x, z)=z$. Since we assumed that $\Omega$ is a ball, $\xi_{j}(x, z) \in \Omega$ whenever $x, z \in \Omega$.

We define the manifold $V(x, z)$ as the set of $\left(B^{1}, \ldots, B^{q}\right) \in X$ such that

$$
\left\{\begin{array}{l}
B^{j}\left(z^{j}-x^{j}\right)=F\left(\xi_{j}(x, z)\right)-F\left(\xi_{j-1}(x, z)\right), \\
B^{j} \in \mathscr{S}_{j}
\end{array}\right.
$$

for $j=1, \ldots, q$.

Let us show that $V(x, z) \neq \varnothing$ for all $x, z \in \Omega$. In fact, by (5.2),

$$
\begin{aligned}
F\left(\xi_{j}(x, z)\right)-F\left(\xi_{j-1}(x, z)\right) & \\
= & {\left[\int_{0}^{1} J\left(\xi_{j-1}(x, z)+t\left[\xi_{j}(x, z)-\xi_{j-1}(x, z)\right]\right) d t\right] } \\
& =\left[\xi_{j}(x, z)-\xi_{j-1}(x, z)\right] \\
& {\left[\int_{0}^{1} J_{j}\left(\xi_{j-1}(x, z)+t\left[\xi_{j}(x, z)-\xi_{j-1}(x, z)\right]\right) d t\right]\left(z^{j}-x^{j}\right) . }
\end{aligned}
$$

But, since $\mathscr{S}_{j}$ is a linear manifold,

$$
\tilde{J}_{j}(x, z)=\int_{0}^{1} J_{j}\left(\xi_{j-1}(x, z)+t\left[\xi_{j}(x, z)-\xi_{j-1}(x, z)\right]\right) d t
$$

belongs to $\mathscr{S}_{j}$ and hence, by (5.4),

$$
\left(\widetilde{J}_{1}(x, z), \ldots, \widetilde{J}_{q}(x, z)\right) \in V(x, z) .
$$

With the definition (5.3) for $V(x, z)$, the optimization problem (5.1) is clearly equivalent to setting

$$
\left(B_{k+1}^{1}, \ldots, B_{k+1}^{q}\right)=P_{V\left(x_{k}, x_{k+1}\right)}\left(\left(B_{k}^{1}, \ldots, B_{k}^{q}\right)\right)
$$

and, therefore, GSFD becomes a particular case of the general algorithm presented in $\S 2(2.2-2.5)$.

In the following theorem, we prove local and superlinear convergence of GSFD.

Theorem 5.1. Let $F$ satisfy Assumption 1 of $\S 2$. There exist $\varepsilon, \delta>0$ such that, if $\left|x_{0}-x_{*}\right| \leq \varepsilon$ and $\left|B_{0}-J(*)\right| \leq \delta$, the sequence generated by Algorithm 5.1 is well defined and converges superlinearly to $x_{*}$.

Proof. Define $E_{*}=\left(J_{1}\left(x_{*}\right), \ldots, J_{q}\left(x_{*}\right)\right)$. So, Assumption 2 of $\S 2$ is trivially satisfied with $r_{*}=0$. Assumption 4 is trivial, since $\|\cdot\|_{x z}=\|\cdot\|$ for all $x, z \in \Omega$. Hence, for proving local linear convergence, we only need to verify Assumption 3. 
By (5.5), it is sufficient to prove that there exists $c_{1} \geq 0$ such that

$$
\left\|\left(\tilde{J}_{1}(x, z), \ldots, \tilde{J}_{q}(x, z)\right)-\left(J_{1}\left(x_{*}\right), \ldots, J_{q}\left(x_{*}\right)\right)\right\| \leq c_{1} \sigma(x, z)^{p}
$$

for all $x, z \in \Omega$. But (5.7) is a straightforward consequence of (2.4). Therefore, the convergence of the sequence and (2.9) are proved.

Now, adding the equalities (5.3) for $j=1, \ldots, q$, we obtain

$$
B^{1}\left(z^{1}-x^{1}\right)+\cdots+B^{q}\left(z^{q}-x^{q}\right)=F(z)-F(x)
$$

for all $\left(B^{1}, \ldots, B^{q}\right) \in V(x, z)$. Therefore,

$$
\left(B^{1}, \ldots, B^{q}\right)(z-x)=F(z)-F(x)
$$

and hence,

$$
B_{k+1}\left(x_{k+1}-x_{k}\right)=F\left(x_{k+1}\right)-F\left(x_{k}\right)
$$

for all $k=0,1,2, \ldots$.

Therefore, by (2.4),

$$
\begin{aligned}
\lim _{k \rightarrow \infty} & \frac{\left|\left[\varphi\left(x_{k+1}, E_{k+1}\right)-\varphi\left(x_{*}, E_{*}\right)\right]\left(x_{k+1}-x_{k}\right)\right|}{\left|x_{k+1}-x_{k}\right|} \\
& =\lim _{k \rightarrow \infty} \frac{\left|F\left(x_{k+1}\right)-F\left(x_{k}\right)-J\left(x_{*}\right)\left(x_{k+1}-x_{k}\right)\right|}{\left|x_{k+1}-x_{k}\right|}=0 .
\end{aligned}
$$

Thus, by Theorem 2.2 , superlinear convergence is proved.

Remarks. The reason why Theorem 5.1 cannot be proved using the DennisWalker theory is easy to understand. The manifold $V(x, z)$ cannot be characterized as the intersection of $Q(F(z)-F(x), z-x)$ with a fixed manifold $\mathscr{A}$. In fact, $V(x, z) \subset Q(F(z)-F(x), z-x)$, but the elements of $V(x, z)$ satisfy additional auxiliary secant equations, which represent a variable linear manifold close to $J\left(x_{*}\right)$.

The CPR property which is used in the SFD algorithm of Dennis and $\mathrm{Li}$ essentially says that for $j=2, \ldots, q$ there exists only one matrix in the set defined by (5.3). This point suggests a potentially useful generalization of the CPR property. Let us say that $\pi_{j}$ satisfies the "CPR $(\nu)$-property," $0 \leq \nu \leq n$, if $n-\nu$ rows of $B^{j}$ are completely determined by (5.3). Clearly, if $\nu$ is large, we may find partitions with a lower $q$. We do not know at what point the practical performance of GSFD deteriorates when $\nu$ grows, but some numerical study in this direction would be worthwhile.

\section{Generalization of the Hart-Soul algorithm}

Hart and Soul [24] introduced an algorithm for solving nonlinear systems of equations which arise from the discretization of nonlinear boundary problems. Essentially, they observed that for this class of problems the Jacobian matrix has the form

$$
J(x)=M_{2}+D_{1}(x) M_{1}+D_{0}(x),
$$

where $M_{2}$ and $M_{1}$ are fixed matrices related to the discretization of second and first derivatives respectively, and $D_{1}(x)$ and $D_{0}(x)$ are diagonal matrices difficult to compute. 
Consequently, Hart and Soul proposed a quasi-Newton method, which consists of the iteration

$$
x_{k+1}=x_{k}-B_{k}^{-1} F\left(x_{k}\right)
$$

where

$$
B_{k}=M_{2}+C_{1, k} M_{1}+C_{0, k} M_{0}
$$

for $k=0,1,2, \ldots$ Here, $C_{1, k}$ and $C_{0, k}$ are diagonal matrices, and $C_{1, k+1}$ and $C_{0, k+1}$ are obtained using a secant equation and a variational principle. Kelley and Sachs [26] considered an infinite-dimensional extension of the HartSoul method and proved superlinear convergence in that context.

The generalization of the Hart-Soul method which we propose in this section applies to functions such that the Jacobian matrix has the form

$$
J(x)=M_{m}+\sum_{j=1}^{m-1} D_{j}(x) M_{j}+D_{0}(x),
$$

where $M_{1}, \ldots, M_{m}$ are fixed matrices (generally originating from discretizations of differential operators) and $D_{0}(x), \ldots, D_{m-1}(x)$ are diagonal matrices. The Hart-Soul case corresponds to $m=2$.

As in the Hart-Soul method, we use the iteration (6.2), but we impose that

$$
B_{k}=M_{m}+\sum_{j=1}^{m-1} C_{j, k} M_{j}+C_{0, k},
$$

where $C_{0, k}, \ldots, C_{m-1, k}$ are diagonal matrices. The matrices $C_{0, k+1}, \ldots$, $C_{m-1, k+1}$ are chosen so as to solve the following optimization problem:

$$
\begin{aligned}
& \text { Minimize }\left\|C_{0}-C_{0, k}\right\|_{F}^{2}+\cdots+\left\|C_{m-1}-C_{m-1, k}\right\|_{F}^{2} \\
& \text { s.t. }\left(M_{m}+\sum_{j=1}^{m-1} C_{j} M_{j}+C_{0}\right)\left(\begin{array}{c}
\left.x_{k+1}-x_{k}\right)=F\left(x_{k+1}\right)-F\left(x_{k}\right), \\
C_{0}, C_{1}, \ldots, C_{m-1} \text { diagonal. }
\end{array}\right.
\end{aligned}
$$

We call the algorithm defined by (6.2), (6.5), and (6.6) the Generalized HartSoul (GHS) method.

Let us show that the GHS method may be considered a particular case of (2.2)-(2.5). We define $X=\mathbb{R}^{n \times n} \times \cdots \times \mathbb{R}^{n \times n}=\mathbb{R}^{n \times n \times m}$. For $\left(C_{0}, \ldots, C_{m-1}\right) \in$ $X$, define $\left\|\left(C_{0}, \ldots, C_{m-1}\right)\right\|=\left(\left\|C_{0}\right\|_{F}^{2}+\cdots+\left\|C_{m-1}\right\|_{F}^{2}\right)^{1 / 2}$ and $\|\cdot\|_{x z}=\|\cdot\|$ for all $x, z \in \Omega$. If $x, z \in \Omega$, we define

$$
\begin{gathered}
V(x, z)=\left\{\left(C_{0}, \ldots, C_{m-1}\right) \in X \mid C_{0}, \ldots, C_{m-1}\right. \text { are diagonal and } \\
\left.\left[M_{m}+\sum_{j=1}^{m-1} C_{j} M_{j}+C_{0}\right](z-x)=F(z)-F(x)\right\} .
\end{gathered}
$$


Now,

$$
\begin{aligned}
F(z)-F(x)= & {\left[\int_{0}^{1} J(x+t(z-x)) d t\right](z-x) } \\
= & \left(\int _ { 0 } ^ { 1 } \left[M_{m}+\sum_{j=0}^{m-1} D_{j}(x+t(z-x)) M_{j}\right.\right. \\
& \left.\left.\quad+D_{0}(x+t(z-x))\right] d t\right)(z-x) .
\end{aligned}
$$

Therefore,

$$
\left(\int_{0}^{1} D_{0}(x+t(z-x)) d t, \ldots, \int_{0}^{1} D_{m-1}(x+t(z-x)) d t\right) \in V(x, z),
$$

and hence $V(x, z)$ is nonempty.

Since $\|\cdot\|$ is obviously associated with a scalar product on $X$, the GHS method has the form (2.2)-(2.3).

To prove local superlinear convergence of the algorithm, assume that, for all $x \in \Omega$ and $j=0,1, \ldots, m-1$,

$$
\left|D_{j}(x)-D_{j}\left(x_{*}\right)\right| \leq L^{\prime}\left|x_{j}-x_{*}\right|^{p} .
$$

Theorem 6.1. Let $F$ satisfy Assumption 1 and (6.10). There exist $\varepsilon, \delta>0$ such that, if $\left|x_{0}-x_{*}\right| \leq \varepsilon$ and $\left|C_{j, 0}-D_{j}\left(x_{*}\right)\right| \leq \delta, j=0,1, \ldots, m-1$, the sequence generated by (6.2), (6.5), and (6.6) is well defined and converges superlinearly to $x_{*}$.

Proof. If $E_{*}=\left(C_{0}\left(x_{*}\right), \ldots, C_{m-1}\left(x_{*}\right)\right)$, Assumption 2 of $\S 2$ is trivially satisfied with $r_{*}=0$. Assumption 3 is a straightforward consequence of (6.9) and (6.10), and Assumption 4 is trivial. Therefore, by an application of Theorems 2.1 and 2.2 , the desired result is proved.

\section{ACKNOWLEDGMENT}

The author is indebted to two anonymous referees for helpful comments.

\section{BIBLIOGRAPHY}

1. C. G. Broyden, A class of methods for solving nonlinear simultaneous equations, Math. Comp. 19 (1965), 577-593.

2. __ A new double-rank minimization algorithm, Notices Amer. Math. Soc. 16 (1969), 670.

3. The convergence of an algorithm for solving sparse nonlinear systems, Math. Comp. 25 (1971), 285-294.

4. C. G. Broyden, J. E. Dennis, and J. J. Moré, On the local and superlinear convergence of quasi-Newton methods, J. Inst. Math. Appl. 12 (1973), 223-245.

5. F. F. Chadee, Sparse quasi-Newton methods and the continuation problem, TR SOL No. 85-8, Dept. of Operations Research, Stanford University, 1985.

6. T. F. Coleman and J. J. Moré, Estimation of sparse Jacobian matrices and graph coloring problems, SIAM J. Numer. Anal. 8 (1983), 639-655.

7. A. M. Curtis, M. J. D. Powell, and J. K. Reid, On the estimation of sparse Jacobian matrices, J. Inst. Math. Appl. 13 (1974), 117-120. 
8. W. C. Davidon, Variable metric methods for minimization, Argonne National Laboratory Report ANL-5990, 1959.

9. J. E. Dennis, Jr., Toward a unified convergence theory for Newton-like methods, Nonlinear Functional Analysis and Applications (L. B. Rall, ed.), Academic Press, New York, 1971, pp. 425-472.

10. J. E. Dennis, Jr., D. M. Gay, and R. E. Welsch, An adaptive nonlinear least-squares algorithm, ACM Trans. Math. Software 7 (1981), 348-368.

11. J. E. Dennis, Jr. and G. Li, A hybrid algorithm for solving sparse nonlinear systems of equations, Math. Comp. 50 (1988), 155-166.

12. J. E. Dennis, Jr. and J. J. Moré, A characterization of superlinear convergence and its application to quasi-Newton methods. Math. Comp. 28 (1974), 549-560.

13. J. E. Dennis, Jr. and R. B. Schnabel, Least change secant updates for quasi-Newton methods, SIAM Rev. 21 (1979), 443-459.

14. _ Numerical methods for unconstrained optimization and nonlinear equations, PrenticeHall, Englewood Cliffs, NJ, 1983.

15. J. E. Dennis, Jr. and H. F. Walker, Convergence theorems for least-change secant update methods, SIAM J. Numer. Anal. 18 (1981), 949-987.

16. R. Fletcher, $A$ new approach to variable metric algorithms, Comput. J. 13 (1970), 317-322.

17. R. Fletcher and M. J. D. Powell, A rapidly convergent descent method for minimization, Comput. J. 6 (1963), 163-168.

18. D. Goldfarb, A family of variable metric methods derived by variational means, Math. Comp. 24 (1970), 23-26.

19. J. L. Greenstadt, Variations on variable metric methods, Math. Comp. 24 (1970), 1-22.

20. A. Griewank and $\mathrm{Ph}$. L. Toint, On the unconstrained optimization of partially separable functions, Nonlinear Optimization 1981 (M. J. D. Powell, ed.), Academic Press, New York, 1982.

21. _ Partitioned variable metric for large structured optimization problems, Numer. Math. 39 (1982), 119-137.

22. __ Local convergence analysis for partitioned quasi-Newton updates, Numer. Math. 39 (1982), 429-448.

23. _ Numerical experiments with partially separable optimization problems, Numerical Analysis Proceedings, Dundee 1983 (D. F. Griffiths, ed.), Lecture Notes in Math., vol. 1066, Springer-Verlag, Berlin, 1984, pp. 203-220.

24. W. E. Hart and S. O. W. Soul, Quasi-Newton methods for discretized nonlinear boundary problems, J. Inst. Math. Appl. 11 (1973), 351-359.

25. G. W. Johnson and N. H. Austria, A quasi-Newton method employing direct secant updates of matrix factorizations, SIAM J. Numer. Anal. 20 (1983), 315-325.

26. C. T. Kelley and E. W. Sachs, A quasi-Newton method for elliptic boundary value problems, SIAM J. Numer. Anal. 24 (1987), 516-531.

27. J. M. Martínez, A family of quasi-Newton methods for nonlinear equations with direct secant updates of matrix factorizations, SIAM J. Numer. Anal. 27 (1990), 1034-1049.

28. __ Local convergence theory of inexact Newton methods based on structured least change updates, Math. Comp. 55 (1990), 143-167.

29. E. S. Marwil, Exploiting sparsity in Newton-type methods, Cornell Applied Mathematics Ph.D. Thesis, Cornell University, Ithaca, NY, 1978.

30. J. M. Ortega and W. C. Rheinboldt, Iterative solution of nonlinear equations in several variables, Academic Press, New York, 1970.

31. M. J. D. Powell, A new algorithm for unconstrained optimization, Nonlinear Programming (J. B. Rosen, O. L. Mangasarian, and K. Ritter, eds.), Academic Press, New York, 1970, pp. 31-65.

32. L. K. Schubert, Modification of a quasi-Newton method for nonlinear equations with a sparse Jacobian, Math. Comp. 24 (1970), 27-30. 
33. D. F. Shanno, Conditioning of quasi-Newton methods for function minimization, Math. Comp. 24 (1970), 647-657.

34. $\mathrm{Ph}$. L. Toint, On sparse and symmetric matrix updating subject to a linear equation, Math. Comp. 31 (1977), 954-961.

35. __ Numerical solution of large sets of algebraic nonlinear equations, Math. Comp. 46 (1986), 175-189.

Applied Mathematics laboratory, IMECC-UNiCAMP, CP 6065, 13081 Campinas SP, BRAZIL

E-mail address: martinez@ccvax.unicamp.ansp.br 